\title{
真空退火超导材料 $\mathrm{Ba}_{2} \mathrm{Y}_{1} \mathrm{Cu}_{3} \mathrm{O}_{7-\delta}$ 的 光电子能谱研究 ${ }^{*}$
}

\author{
胡典文 伍乃娟 沈电洪齐上雪 谢 \\ (中国科学院物理研究所,北京)
}

关虂词高 $T_{\mathrm{c}}$ 超导体、光电子能谱、真空退火、 $\mathrm{Ba}_{2} \mathrm{Y}_{1} \mathrm{Cu}_{3} \mathrm{O}_{7-8}$

\section{一、引}

Ba- -Y - Cu-O 材料具有液氮温区超导特性的发现 ${ }^{[1,2]}$, 使人们对此发生了极大兴趣。这种 超导材料的性能与烧结温度和烧结气氛有关. 事实上, 不同的烧结和退火条件将严重影响 样品的氧含量. $\mathrm{X}$ 射线衍射照相及中子衍射 ${ }^{[3,4]}$ 研究表明, 具有畸变钙钛矿结构的超导材料 $\mathrm{Ba}_{2} \mathrm{Y}_{1} \mathrm{Cu}_{3} \mathrm{O}_{7-8}$ 中氧空位起重要作用. 由于超导特性根本上是由材料的电子结构特性决定的, 因此不同退火条件下,这些材料的电子能态的变化及其影响是需要认真研究的.

Shin-iChi Shamoto 等人对超导材料 $\mathrm{La}-\mathrm{Sr}-\mathrm{Cu}-\mathrm{O}$ 进引了真空退火材料超导特性实 验影. 实验表明当退火温度小于 $600^{\circ} \mathrm{C}$ 时, $T_{\mathrm{c}}$ 转变温度基本不变, 而退火温度高至 $900^{\circ} \mathrm{C}$ 时, 样品不 再显示超导特性。

Massidda 等 ${ }^{[6]}$ 和 Mattheiss 等 ${ }^{[7]}$ 分别计算了 $\mathrm{Ba}_{2} \mathrm{Y}_{1} \mathrm{Cu}_{3} \mathrm{O}_{7-8}$ 超导材料的能带结构. 他们 的研究结果表明, $E_{F}$ 附近的态密度对 $\delta$ 的大小很敏感. 当 $\delta$ 增大时, 即氧空位较多时, $E_{F}$ 附 近态密度将下降。

我们在高真空中对 $\mathrm{Ba}_{2} \mathrm{Y}_{1} \mathrm{Cu}_{3} \mathrm{O}_{7-8}$ 超导样品进行了高温退火实验, 并用 XPS 研究样品在 各情形下电子价态结构的变化, 讨论了氧的释放以及各金属离子还原特性和电子逐渐局域化 的过程.

\section{二、实 验}

样品 $\mathrm{Ba}_{2} \mathrm{Y}_{1} \mathrm{Cu}_{3} \mathrm{O}_{7-8}$ 是用 $\mathrm{BaCO}_{3} 、 \mathrm{Y}_{2} \mathrm{O}_{3}$ 和 $\mathrm{CuO}$ 粉末压成合适样品台大小的圆片状, 在高 温下烧结而成。样品在 $92 \mathrm{~K}$ 起始超导转变, 在 $88 \mathrm{~K}$ 处达零电阻. $\mathrm{X}$ 射线粉末衍射分析表明 样品杂相很少. XPS 实验在表面分析仪 VGESCALAB5 上进行. 预处理室的本底真空度为 $1 \times 10^{-7} \mathrm{~Pa}$, 主真空室真空度为 $4 \times 10^{-8} \mathrm{~Pa}$ 。样品先在预处理室用氧化铝刀口摩擦表面以除 去污染, 在处理后样品的 XPS 谱峰中碳 $1 s$ 峰仅勉强能分辨. 然后在预处理室对样品进行真 空退火, 再传递样品到主真空室进行能谱测量. 在退火过程中, 由于篻从样品中逸出, 预处理 室真空可下降到 $10^{-5}-10^{-4} \mathrm{~Pa}$ 。 实验中 $\mathrm{X}$ 射线光源用 $\mathrm{AlK}_{a}(1486.6 \mathrm{eV})$.

通过计算机处理将实验中记录的能谱按各元素的主峰高度归一, 因此不同谱峰强度不表

本文 1987 年 8 月 12 日收到.

- 国家自然科学基金资助项目. 
示相对含量,只有同一㙕峰的相对强度才有意义。

\section{三、结果和 讨 论}

r仔细的测量已表明，尽管样品在超高真空中会放氧，在室温中采用小 X射线束流密度经过 $2 \mathrm{~h}$ 的测量, 光电子谱蜂分布基本不变. 然而, 若将样品加热退火, 样品能谱发生明显变化, $\mathrm{O}$ 峰强度相对其它谱峰降低,表明这是一个放氧过程. 图 1 则给出随退火温度的升高, O1s XPS 谱峰的结构变化 (已对主峰高度归一). 015 峰明显呈双峰结构.作者在另一文中 ${ }^{[8]}$, 分析了一 系列超导样品 $\mathrm{Ba}_{1-x} \mathrm{Y}_{x} \mathrm{CuO}_{3-y}(x$ 从 0.1 到 0.6 ) 的 $\mathrm{O} 1 \mathrm{~s}$ 峰变化规律,表明双峰中低结合能端的

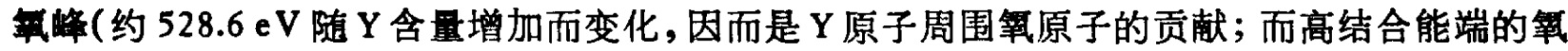
峰 (约 $531.5 \mathrm{eV}$ ) 是晶格中其余氧原子的贡献，在退火温度升高的初始阶段 $\left(400^{\circ} \mathrm{C}\right.$ 以下，见图 $1 \mathrm{a}-\mathrm{c})$, 高结合能端的氧峰略为相对增强, 表明此时 $\mathrm{Y}$ 周围的氧相对有较多的释放。虽然一 系列结构研究表明这类羍化物在 $\mathrm{Ba}$ 原子层之间的 $\mathrm{CuO}_{6}$ 底平面上容易形成篻空位 ${ }^{[3,4]}$, 然而 可能在真空退火的初始阶段 (小于 $400{ }^{\circ} \mathrm{C}$ ), 脱附的氧原子在 $\mathrm{Ba}_{2} \mathrm{Y}_{1} \mathrm{Cu}_{3} \mathrm{O}_{7-8}$ 中氧空位上迁移,平 均效果没有给出明显可观测的这种光电子能谱变化。当退火温度升至 $550^{\circ} \mathrm{C}$ 后（见图 $1 \mathrm{~d}, \mathrm{e}$ ), 高结合能端篻蜂明显减小。可见此时 $\mathrm{BaO}$ 层和 $\mathrm{Ba}$ 层之间的 $\mathrm{CuO}_{6}$ 平面中的氧原子相对释出 较多. 这一过程继续进行, 直至样品经 $800^{\circ} \mathrm{C}$ 退火 $15 \mathrm{~min}$ 后, 氧峰变得很宽. 由 X 射线衍射分 析表明经 $800{ }^{\circ} \mathrm{C}$ 退火的样品中存在许多杂相. 因不同结构相中 $01 \mathrm{~s}$ 可能有不同的化学移位, 圣加的结果导致 $01 s$ 谱峰的加宽.

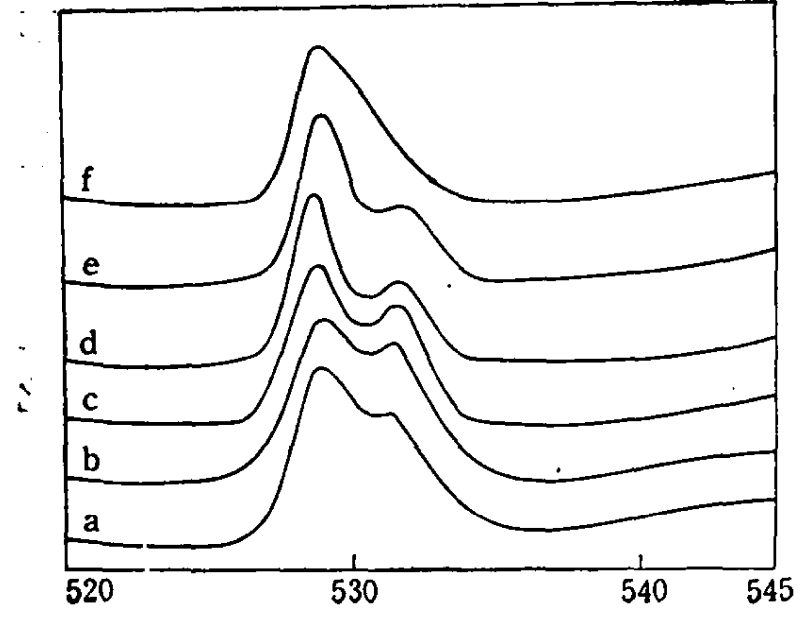

图 $1 \mathrm{Ba}_{2} \mathrm{Y}_{1} \mathrm{Cu}_{3} \mathrm{O}_{-6}$ 中 $\mathrm{O} 1$ s 谱略随真 空退火温度的变化

e. 察温; b. $200^{\circ} \mathrm{C}, 15 \mathrm{~min} ; \mathrm{c.} 400^{\circ} \mathrm{C}, 15 \mathrm{~min} ; \mathrm{d} .550^{\circ} \mathrm{C}$, $15 \mathrm{~min}$; e. $650^{\circ} \mathrm{C}, 15 \mathrm{~min}, \mathrm{f.} 80^{\circ} \mathrm{C}, 15 \mathrm{~min}$

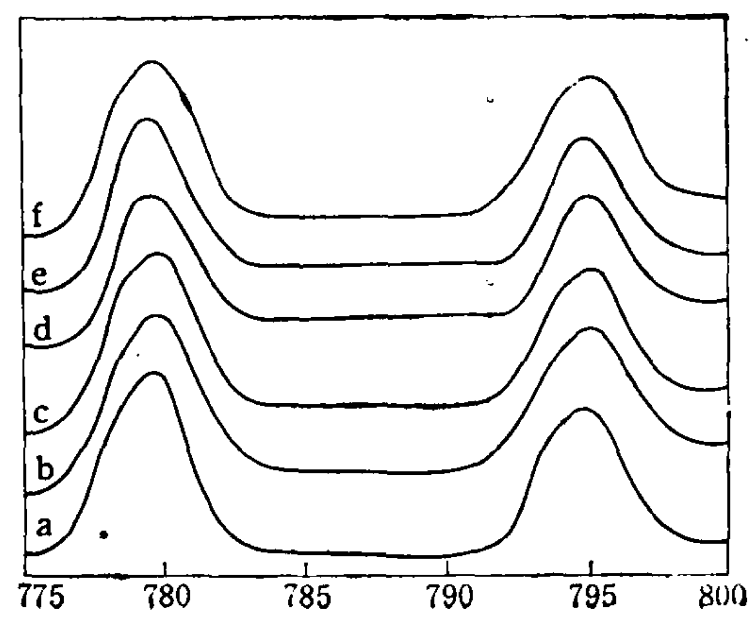

图 $2 \mathrm{Ba}_{2} \mathrm{Y}_{1} \mathrm{Cu}_{3} \mathrm{O}_{1-8}$ 中 $\mathrm{Ba} 3 d$ 谱峰随真 空退火温度的变化

8. 室温; b. $200^{\circ} \mathrm{C}, 15 \mathrm{~min}$; c. $400^{\circ} \mathrm{C}, 15 \mathrm{~min}$; d. $550^{\circ} \mathrm{C}$, $15 \mathrm{~min}$; e.650 $\mathrm{C}$, $15 \mathrm{~min} ; \mathrm{f.} 80^{\circ} \mathrm{C}, 15 \mathrm{~min}$

在室温下, $\mathrm{Ba}_{2} \mathrm{Y}_{1} \mathrm{Cu}_{3} \mathrm{O}_{7-8}$ 中 $\mathrm{Ba}_{3} d_{5 / 2}$ 和 $\mathrm{Ba}_{3} d_{3 / 2}$ 呈双蜂结构 (见图 2)。低结合能端的 $\mathrm{Ba} 3 d$ 峰 (如 $\mathrm{Ba} 3 d_{3 / 2}$ 中约 $778 \mathrm{eV}$ 处谱峰) 与 $\mathrm{Y}$ 原子的存在有关 ${ }^{[8]}$. 事实上由于晶格中 $\mathrm{Y}^{3+}$ 代 替 $\mathrm{Ba}^{2+}$ 改变了年在晶格中的状态,并通过 $\mathrm{Y}$ 周围的篻降低了部分 $\mathrm{Ba}$ 的离化价. 正如 $\mathrm{O} 1 s$ 谱的 变化表明, 在真空退火的初始阶段(图 $2 \mathrm{a}-\mathrm{c}$ ), 由于 $\mathrm{Y}$ 周围有少量篻释出，而 $\mathrm{Y}^{3+}$ 与余下的 $\mathrm{O}^{2-}$ 有较强的相互作用, 这进一步增加了 $\mathrm{Ba}$ 的还原, 因此 $\mathrm{Ba}$ 低结合能端蜂有所增加. 当退火 温度升至 $550^{\circ} \mathrm{C}$ 后(图 2d, e)，由于 $\mathrm{Ba}$ 层之间的 $\mathrm{CuO}_{6}$ 和 $\mathrm{BaO}$ 层中氧的释出, $\mathrm{Ba} 3 d$ 峰宽 
逐渐变窄,这可能是因为 $\mathrm{Ba}$ 周围篻的析出, 使 $\mathrm{Ba}$ 周围的环境差别反而变小了. 当经 $800{ }^{\circ} \mathrm{C}$ 莩 火 $15 \mathrm{~min}$ 后, Ba3d 峰宽反而增加. 正如前述, 此时晶体结构发生较大变化, 多相化物中 Ba 原子周围环境差别较大, 造成峰宽增加。

图 3 是 $\mathrm{Y} 3 d$ 峰的变化情况. 真空退火的结果使 $\mathrm{Y} 3 d_{5 / 2}$ 和 $\mathrm{Y} 3 d_{3 / 2}$ 的相对强度发生了变 化. 在低于 $250^{\circ} \mathrm{C}$ 下退火, 样品中的 $\mathrm{Y}$ 谱峰没有明显的变化; 在 $250{ }^{\circ} \mathrm{C}-400^{\circ} \mathrm{C}$ 阶段, 篻的释放 明显地影响 $\mathrm{Y}$ 的化学状态, 两峰变得尖锐, 特别是低能端 $\mathrm{Y} 3 d$ 峰相对增强, 这显示在退火中 钎的还原趋势; 在 $400^{\circ} \mathrm{C}$ 至 $650^{\circ} \mathrm{C}$ 阶段, Y $3 d$ 谱峰基本保持不变; 同样, 在经过 $8000^{\circ} \mathrm{C}$ 退火 $15 \mathrm{~min}$ 后,由于许多杂相的存在,造成 $\mathrm{Y} 3 d$ 峰的变宽.

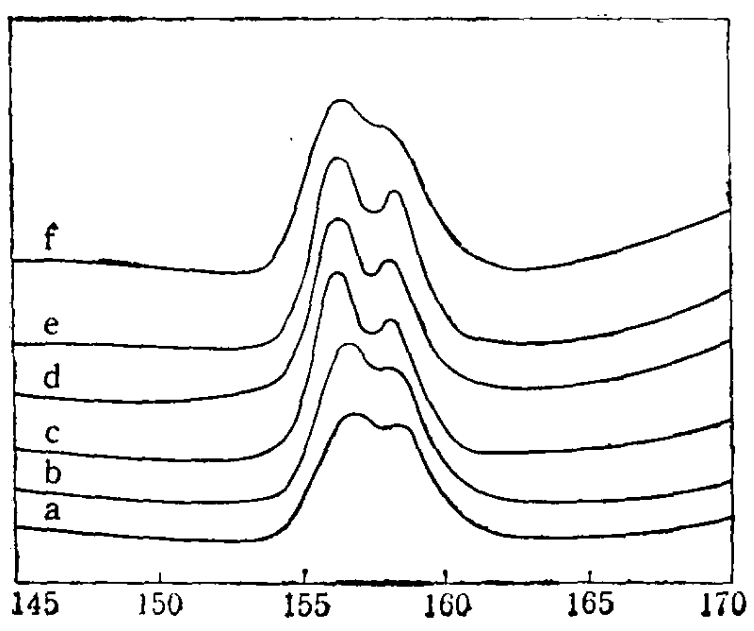

图 $3 \mathrm{Ba}_{2} \mathrm{Y}_{1} \mathrm{Cu}_{3} \mathrm{O}_{7}$ 。中 $\mathrm{Y} 3 d$ 谱峰随真空 退火温度的变化

8. 室温; b. $200^{\circ} \mathrm{C}, 15 \mathrm{~min} ; \mathrm{c.} 400^{\circ} \mathrm{C}, 15 \mathrm{~min}$; d. $550^{\circ} \mathrm{C}, 15 \mathrm{~min}$; e. $650^{\circ} \mathrm{C}, 15 \mathrm{~min} ; \mathrm{f} .800^{\circ} \mathrm{C}, 15 \mathrm{~min}$

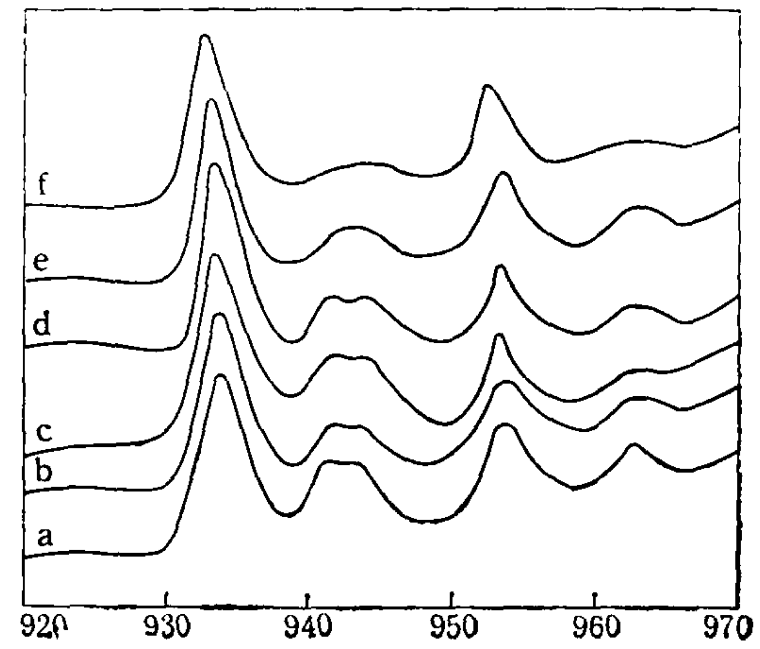

图 $4 \mathrm{Ba}_{2} \mathrm{Y}_{1} \mathrm{Cu}_{3} \mathrm{O}_{r_{-}}$中 中 $\mathrm{Cu}_{2} P$ 谱峰随 真空退火温度的变化

a. 室温; b. $200^{\circ} \mathrm{C}, 15 \mathrm{~min} ;$ c. $400^{\circ} \mathrm{C}, 15 \mathrm{~min} ; \mathrm{d} .550^{\circ} \mathrm{C}$, $15 \mathrm{~min}$; e.650 $0^{\circ} \mathrm{C}, 15 \mathrm{~min}$; f. $800^{\circ} \mathrm{C}, 15 \mathrm{~min}$

图 4 给出了 $\mathrm{Cu} 2 p$ 谱峰相应的变化. 对应于 $\mathrm{Cu} 2 p_{3 / 2}$ 和 $\mathrm{Cu} 2 p_{1 / 2}$ 的两卫星峰是光电子 非弹性散射过程中, 电子由 $\mathrm{O} 2 p$ 态向 Cu3d 空电子态跃迁而产生的. 随着退火温度的升高, 这两个卫星峰相对于主峰的强度逐渐减小,并且主峰位置逐渐向低结合能端移位,表明篻含量 逐渐减少, $\mathrm{Cu}$ 离子逐渐还原。在经 $800^{\circ} \mathrm{C}$ 高温退火 $15 \mathrm{~min}$ 后, 铜的价态中已有大量的 $\mathrm{Cu}^{+1}$. 需要说明的是, $\mathrm{Cu} 2 p$ 的能谱变化并没有象 $\mathrm{O} 1 s, \mathrm{Ba} 3 d$ 和 $\mathrm{Y} 3 d$ 那样,给出随温度变化的阶段 特点,这可能是因为 $\mathrm{Cu}$ 在晶格中有两种不同的位置，氧的释出对它都有影响，而给出总的还 原特性。

\section{四、小结}

在真空中对 $\mathrm{Ba}_{2} \mathrm{Y}_{1} \mathrm{Cu}_{3} \mathrm{O}_{7-\delta}$ 超导材料进行高温退火,样品光电子能谱的变化清楚表明这是 一个放篻过程. $\mathrm{Y}$ 原子周围氧的释出使得 $\mathrm{Y} 3 d$ 和 $\mathrm{Ba}$ 3d 光电子能谱有较大的变化. 而 $\mathrm{BaO}$ 平面和 $\mathrm{Ba}$ 层之间的 $\mathrm{CuO}_{6}$ 平面上的氧的大量释出, 导致 $\mathrm{Cu}$ 离子的逐渐还原. 在约 $800{ }^{\circ} \mathrm{C}$ 温区, 羊在各位置的大量释出, 使得钻钢矿结构骨架出现崩溃, 导致许多杂相, 由于无序的羍空 位的增加和结构的杂乱性, 样品最终退化为非超导材料. 此外, 紫外光电子能谱 (UPS) 测量 也表明,由于篻、铜及其耦合在 $0-1.2 \mathrm{eV}$ 的价态峰,在低于 $600{ }^{\circ} \mathrm{C}$ 退火中变化不大, $2.5 \mathrm{eV}$ 的 
Cu3d 谱蜂逐渐增强，铜逐渐还原，但样品仍保持超导特性，然而加温到 $800{ }^{\circ} \mathrm{C} 15 \mathrm{~min}$ 后，0$1.2 \mathrm{eV}$ 的峰消失,出现禁带, 电子局域化,样品呈绝缘性. 当然,还需要更加细致的实验来决定 控制晶格中筒的条件一晶格结构一电子局域化的确切关系, 以及这一关系在体相和表面相的差 别，这些知识对确定氧含量与样品电子结构的关系以及提高样品的临界电流有重要的参考意 义。

\section{文文}

[1] 忠贤、陈立家等，科学通报，32(1987)，3：177-179.

[ 2 ] Wu, M. K., Ashburn, J. R. et al., Phys. Rev. Lezzers, 58(1987), 908.

[3] Beno, M. A., Soderholm, L. et al., Submitted to Appl. Phys. Letzes.

[4] Yan, Q. W. et al., Submitted to Phys. Rev. Letters.

[5] Shin-ichi Shamoto et al. (in press).

[6] Massidda, S. Yu Jaejun and Freeman, A. J, Phys. Rev. Lezters, 122(1987), 198.

[ 7 ] Mattheiss, L. F. and Hammann, D. R., Submitted toolid State Commun.

[8]伍乃㛎、胡典文、沈电烘、齐上至、周越、郡妯瑜，科学通报，33(1988)，14: 1057-1060. 